\title{
Phytotherapie bei Erkrankungen der Leber und Galle
}

Hartmut Dorstewitz

Von unseren inneren Organen ist die Leber das größte und wiegt etwa $1,5-2 \mathrm{~kg}$. Sie wird täglich von etwa 2000 Litern Blut durchflossen, das sind etwa 4 Blutkonserven pro Minute. Über die Pfortader nimmt sie nährstoffhaltiges Blut (z.B. Fette, Kohlenhydrate, Eiweiße, Glukose, Vitamine und Spurenelemente) aus dem Intestinaltrakt auf, um sie zu verstoffwechseln und/ oder zu speichern. Zudem werden Toxine (exogene Schadstoffe und endogene Abbauprodukte) in der Leber gebunden und in eine ausscheidungsfähige Form gebracht. Eine weitere Aufgabe der Leber ist die tägliche Produktion von etwa einem halben Liter Gallensaft, der in der Gallenblase eingedickt und bedarfsweise an den Darm zur Fettverdauung abgeben wird.

Ist der Input an Toxinen in die Leber höher als die Outputleistung der konjugierten Giftstoffe, die durch die Biotransformation in das größte unserer Stoffwechselorgane gelangt sind, dann kommt es, abhängig von der Dauer und dem Grad der Intoxikation zu einer allmählichen Schädigung der Leberzellstruktur.

\section{Mariendistel}

Hier setzt nun eine der wichtigsten Heilpflanzen für die Leber an: die Mariendistel (Silybum marianum oder Carduus marianus). Ihren Namen soll sie daher haben, das der heiligen Maria beim Stillen ein paar Tropfen ihrer Milch auf das Blatt gefallen sind und dadurch das Blatt seine marmorierte Farbe erhielt. Die Bezeich-

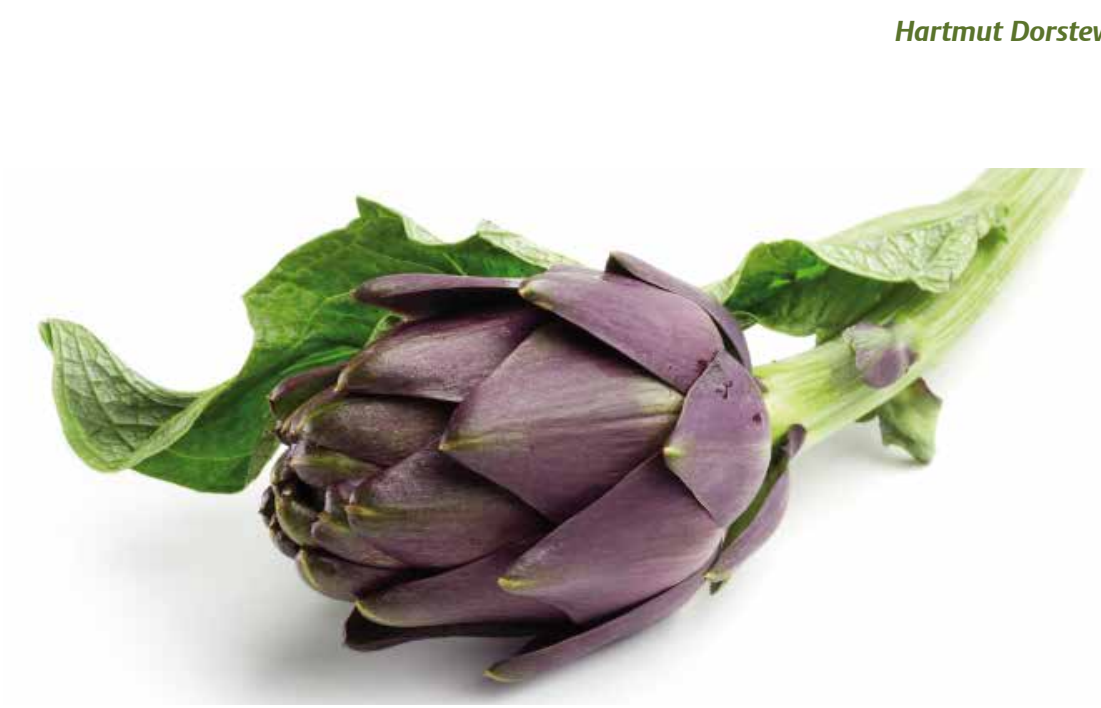

Abb. 1 Cynara scolymus - die Artischocke. @ Paulista/Fotolia

nung Silybum geht auf das griechische Wort Sillybon zurück, was Quaste oder Anhängsel bedeutet und sich wohl auf die Wurzel bezieht oder auf die Blüte.

Sie gehört zu den Korbblütlern (Asteraceae) und zur Unterfamilie der Carduoideae. Wie ihr Name verrät, ist sie eine Distel und zählt zu den am besten untersuchten Heilpflanzen. Ihre Heimat ist ursprünglich der Mittelmeerraum. In unseren Breitengraden kommt sie nur gelegentlich verwildert vor. Verwendet werden die Früchte der Pflanze. Diese enthalten u. a. 1,5-3\% Silymarin. Bei diesem Wirkstoff fand man 3 gut untersuchte Haupteffekte:

- Membraneffekt (leberzellprotektiver Effekt)

- Leberzellregeneration

- Radikalfängerfunktion

Silymarin ist eine Mischung der Isomere von Silibin, Silicristin und Silidianin. Die Substanz stabilisiert die Zellmembran der
Leberzellen. Das hat einen protektiven Effekt auf die Invasion von Zellgiften in das Zellinnere. Offensichtlich werden durch Silymarin Bindungsstellen bzw. Transportsysteme dieser Gifte an der Zellmembran blockiert. Die Antidotwirkung gegenüber den leberzellschädigenden Noxen wie Alkohol, Chemikalien und auch Medikamenten beruht offenbar darauf, dass Silymarin an Zellproteine und Rezeptoren der Zellmembranen binden und dadurch die Toxine verdrängen und ihre Invasion in das Zellinnere vermindern kann.

Zudem fand man eine schützende Wirkung gegen den transmembralen Verlust von Leberzellenzymen. Die zellprotektive Wirkung von Silimarin ist natürlich abhängig von der Höhe der zugeführten Noxen.

Der zellregenerative Effekt erklärt sich durch Aktivierung des Enzyms Polymerase A. Diese steigert die für die Zellintegration wichtige Eiweißsynthese, dazu 
Der Einsatz von Phytotherapeutika bei Erkrankungen der Leber und Galle basiert auf langjährigen guten Erfahrungen in der Praxis, ein Großteil ist von der Kommission $\mathrm{E}$ monografiert. Mariendistel, Artischocke, Rote Bete und Schafgarbe sind bewährte Therapeutika bei Lebererkrankungen. Für Gallenwegsdyskinesien zählen Wermut, Schöllkraut, Erdrauch, Rettich und Löwenzahn zu den wichtigsten Phytotherapeutika. Die Phytotherapie ist eine starke und verlässliche Partnerin in der Behandlung der Leberund Gallenerkrankungen, wenn man sie gezielt einsetzt und sie in ein sinnvolles Therapiekonzept einfügt.

Schlüsselwörter

Lebererkrankung, Gallenwegsdyskinesie, Phytotherapie.

\section{ABSTRACT}

The use of phytotherapeutic agents in the case of diseases of the liver and the gallbladder is based on many years of positive practical experience; the majority is monographed by the Commission E. Milk thistle, artichoke, beetroot, and yarrow are proven therapeutic agents in the case of liver diseases. For biliary dyskinesia wormwood, celandine, fumitory, radish and dandelion belong to the most important phytotherapeutic agents. Phytotherapy is a strong and reliable partner in the treatment of liver and gallbladder diseases, when it is used specifically and embedded into a meaningful therapeutic concept.

\section{Keywords}

Liver disease, biliary dyskinesia, phytotherapy.

onsbehandlung innerhalb von 24 Stunden mit Silybinin (Legalon ${ }^{\circledR}$ SIL; 20 mg pro kg Körpergewicht und Tag in 4 Einzeldosen) kann die Mortalitätsrate auf unter 10\% senken.

Auch bei Leberzirrhose, Fettleber und Fettleberhepatitis fand man eine deutlich verbesserte Überlebensrate. Sogar die Diabetesparameter zeigten eine signifikante Verbesserung.

Klinische Untersuchungen belegen ebenfalls eine antivirale Aktivität von Silibinin gegenüber dem Hepatitis-C-Virus (HCV) und dem Humanen-Immundefizienz-Virus (HIV).

\section{Monografie der Kommission E}

Mariendistelextrakte sind durch die Kommission E positiv monografiert und zur unterstützenden Behandlung bei chronisch entzündlichen Lebererkrankungen und Leberzirrhose geeignet. Die empfohlene Dosierung liegt bei 200-400 mg Silymarin pro Tag. Dabei werden bei oraler Gabe bis zu 20-50\% resorbiert. Die Resorption ist allerdings von der Galenik des jeweiligen Präparats abhängig.

\section{Präparate, Dosierungen, uner- wünschte Wirkungen}

\section{Fertigpräparate und Dosierung}

Gängige Fertigpräparate im Handel sind z.B.:
- Legalon ${ }^{\circledR}$ forte Hartkapseln (Fa. Meda Pharma), 3-mal täglich 1 Hartkapsel unzerkaut mit etwas Flüssigkeit einnehmen

- Legalon $^{\circledR}$ Protect Madaus Hartkapseln (Fa. Meda Pharma), 3-mal täglich 2 Hartkapseln unzerkaut mit etwas Flüssigkeit vor dem Essen einnehmen

- Legalon ${ }^{\circledR}$ SIL Pulver (Fa. Meda Pharma) zur Herstellung einer Infusionslösung

- hepa-loges ${ }^{\circledR}$ Hartkapseln (Fa. Loges), 3-mal täglich 1 Hartkapsel mit ausreichend Flüssigkeit einnehmen

- Hepar-Pasc ${ }^{\circledR}$ (Fa. Pascoe), Erwachsene und Kinder > 12 Jahre: 3 - bis 4-mal täglich 1 Filmtablette unzerkaut mit ausreichend Flüssigkeit einnehmen

- Phytohepar $^{\circledR}$ novo Hartkapseln (Fa. Steigerwald), Erwachsene und Jugendliche ab 12 Jahren: 2-mal täglich 1 Hartkapsel unzerkaut mit reichlich Flüssigkeit jeweils nach einer Mahlzeit einnehmen; Hartkapsel nicht im Liegen einnehmen

\section{Nebenwirkungen}

Nebenwirkungen werden lediglich bei 1-2\% der Fälle beobachtet. In der Regel sind dies nur leichte, passagere gastrointestinale Beschwerden.

\section{Teeanwendung}

Die Mariendistel eignet sich auch als Teeanwendung: Man übergießt 1-2 Teelöffel Mariendistelfrüchte mit 1 Tasse kochendem Wasser und lässt ihn 10-20 Minuten ziehen. Nach dem Abseihen werden täglich 1-3 Tassen schluckweise getrunken. Eine Kur kann wegen der geringen Nebenwirkungen unbedenklich über 6 Wochen und länger angewendet werden.

\section{Artischocke}

Schon die alten Ägypter schätzten die Artischocke (Cynara scolymus) (Abb. 1) als wertvolle Heilpflanze, die sich aber nur die Wohlhabenden leisten konnten. Heute freuen wir uns, wenn sich die wohlschmeckenden und erschwinglichen Artischockenherzen auf unserer Pizza finden. Sie ist ebenfalls ein distelähnlicher Korbblütler (Asteraceae), dessen Heimat das Mittel- 


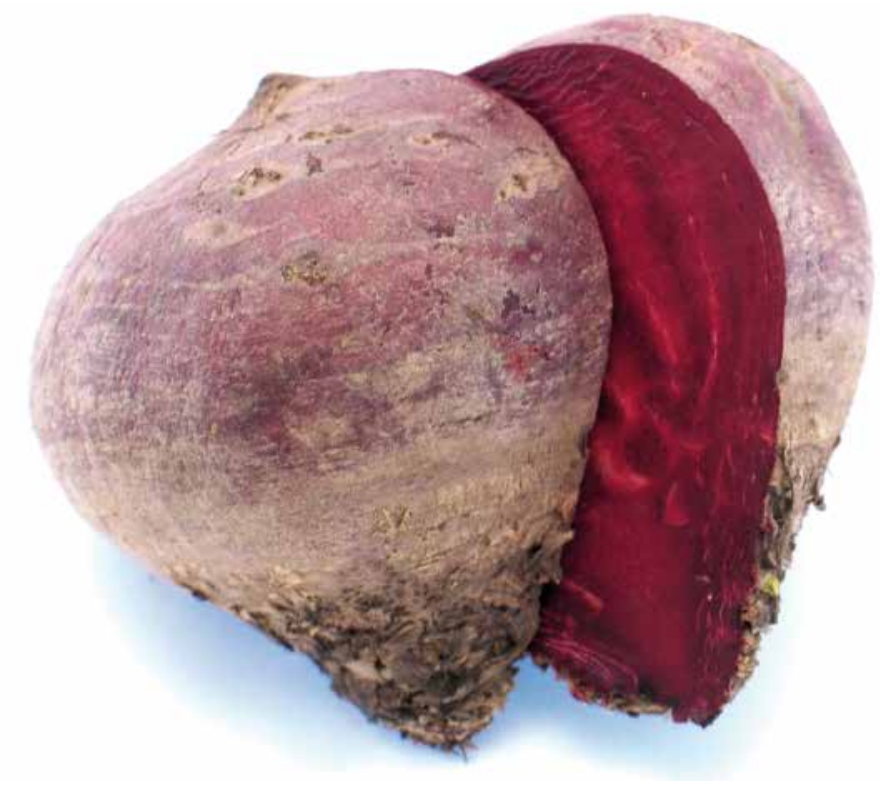

Abb. 2 Beta vulgaris - Rote Bete. @ Lucky Dragon/Fotolia

meergebiet ist. Verwendet werden die Laubblätter (Cynarae folium). Die Artischockenblätter enthalten hauptsächlich 3 Substanzklassen:

- Kaffeesäurederivate (Caffeoylchinasäure), v.a. Chlorogensäure und Cynarin, sowie

- Flavonoide, v.a. Luteolin, und deren Verwandter, das Cynarosid

- und bis zu 4-5\% Bitterstoffe vom Sesquiterpenlactontyp wie das Cynaropikrin.

Ihre Wirkungen sind z.T. ähnlich denen der Mariendistel: leberregenerierend, hepatoprotektiv, antidyspeptisch, karminativ, appetitanregend, antiemetisch, antioxidativ. Dazu kommen noch choleretische und lipidsenkende Effekte.

Cynarin ist für die hepatoprotektive Wirkung verantwortlich. Es schützt die Zellmembran vor Toxinen.

Das Flavonoid Luteolin wirkt antioxidativ, antiphlogistich und immunmodulatorisch. Es hemmt Interleukin 6 und ist ein PDE4-Hemmer. Auch scheint es positive Effekte auf den Kohlenhydratstoffwechsel und eine antikarzinogene Wirkung zu besitzen.

Flavonoide und Caffeoylchinasäure-Abkömmlinge besitzen zudem eine cholagoge und choleretische Wirkung, d.h. sie regen die Gallensäureproduktion in der Leber und deren Abgabe in den Darm an.
Bitterstoffe wie das Cynaropikrin steigern die Magensäureproduktion und regen damit den Appetit an.

Aufgrund synergistischer Wirkmechanismen wie die verminderte Cholesterinsynthese in den Leberzellen, vermehrte Ausscheidung von Cholesterin, erhöhter Cholesterinabbau bei der Gallensäuresynthese senken Artischockenextrakte nach einer 6-wöchigen Anwendungsdauer den Gesamtcholesterin- und Triglyzeridgehalt um bis zu $12 \%$, LDL wird vermehrt in HDL konvertiert.

Die mittlere Tagesdosis wird mit $6 \mathrm{~g}$ Droge angegeben.

\section{Monografie der Kommission E}

Artischockenblätter (Cynarae folium) sind durch die Kommission E positiv monografiert und werden für die Indikation „dyspeptische Beschwerden“ empfohlen.

Als Gegenanzeigen werden Allergie gegen Artischocken oder andere Korbblütler sowie Verschluss der Gallenwege genannt.

Nebenwirkungen und Wechselwirkungen mit anderen Mitteln sind laut Kommission-E-Monografie nicht bekannt.

\section{Präparate und Dosierungen}

Einige im Handel erhältliche Fertigpräparate enthalten standardisierte Artischockenblättertrockenextrakte:

- Ardeycholan ${ }^{\circledast}$ Kps. (Fa. Ardeypharm), Dosierung: Erwachsene und Heran- wachsene ab 12 Jahren: 3-mal 1 Hartkapsel täglich.

- Cholagogum Nattermann ${ }^{\circledR}$ Artischocke Kapseln/Flüssigkeit (Fa. Cassella-med), Dosierung: Zu den Mahlzeiten mit ausreichend Flüssigkeit: Kapseln: Erwachsene und Kinder $>12 \mathrm{~J}$. 3-mal täglich 1 Hartkapsel unzerkaut einnehmen; Liquidum: Erwachsene und Kinder $>12 \mathrm{~J}$. 3 -mal täglich $10 \mathrm{ml}$.

- Hepar-SL ${ }^{\circledR} 320$ mg Hartkapseln (Cassella-med), Hepar-SL ${ }^{\circledR}$ forte $600 \mathrm{mg}$ überzogene Tabletten (Cassella-med), Dosierung: Erwachsene und Heranwachsene ab 12 Jahre nehmen 2-mal täglich 2 Hartkapseln bzw. 1 überzogene Tablette zu den Mahlzeiten unzerkaut mit Flüssigkeit ein.

- Hepar-POS ${ }^{\circledast}$ Hartkapseln (Ursapharm), Dosierung: Erwachsene und Kinder $>12$ Jahre: 3 -mal täglich 1 Hartkps. zu den Mahlzeiten mit Flüssigkeit einnehmen.

\section{Rote Bete}

Ähnliche Wirkung wie die Mariendistel wird der Roten Bete, syn. Rote Rübe (lat. Beta vulgaris) bescheinigt (Abb. 2). Sie ist sehr eng mit der Zuckerrübe und dem Mangold verwandt und eine Kulturform der wilden Rübe und stammt ursprünglich aus dem Mittelmeerraum.

Der Hauptwirkstoff dieser Pflanze ist das Glykosid Betain, das ihr auch die charakteristische rote Farbe verleiht, die blasse Lippen und das Essen auf dem Teller rot färbt. Daneben enthält sie einen hohen Gehalt an Vitamin B, Folsäure, Kalium und Eisen.

Betain gehört, ähnlich wie das Methionin zu den essenziellen hepatotropen Aminosäuren und greift nach Fintelmann und Weiss in der Leberzelle in den Methylierungszyklus ein, indem sie als Mythyldonator wirkt. Das unterstützt die Leberzellregeneration.

Zudem wird der Roten Bete nachgesagt, den Blutdruck und den Homocysteinspiegel zu senken und bei Sportlern eine Leistungssteigerung zu bewirken. Empfohlen wird eine Einnahme von $500 \mathrm{ml}$ Saft täglich bzw. vor der sportlichen Betätigung. 
Präparate und Dosierungen

Betain gibt es als Fertigpräparate im Handel:

- Flacar $^{\circledR}$ (Fa. Schwabe) Beutel, Dosierung: 2- bis 4-mal täglich 1 Beutel

- Cystadane ${ }^{\circledR}$ (Fa. Orphan Europe), Pulver, Dosierung: 2-mal täglich $3 \mathrm{~g}$ Pulver

\section{Schafgarbe}

Keine direkte Wirkung auf den Leberstoffwechsel wohl aber indirekt auf die Oberbauchbeschwerden im Rahmen einer Lebererkrankung kommt der Schafgarbe (Achillea millefolium) zu. Die Schäfer lieben sie, weil sie ihre Schafe gesund macht. Auch Achilles, daher der lateinische Name, kurierte mit ihr seine Wunden. Die Schafgarbe ist eine nahe Verwandte der Kamille (Matricaria chamomilla) und besitzt ähnliche Eigenschaften. Aufgrund ihrer Inhaltsstoffe, ätherische Öle (Proazulen), Bitter- und Gerbstoffe sowie Flavonoide, wirkt sie u.a. spasmolytisch, antiphlogistisch, tonisierend, karminativ, cholagog, appetitanregend und wundheilungsfördernd. Verwendet wird das ganze Kraut (Millefolii herba), vorzugsweise als Teedroge. Da sie zu den häufigsten heimischen Pflanzen gehört, eignet sie sich vorzüglich zur Selbstmedikation: 1 Teelöffel feingeschnittener Droge mit 1 Tasse heißem Wasser übergießen, 5 Minuten abgedeckt ziehen lassen, abseihen, 3- bis 4-mal täglich 1 Tasse langsam schluckweise trinken. Der Tee eignet sich auch sehr gut als feuchtwarmer Leberwickel.

\section{Phytotherapeutika bei Gallenwegsdykinesien}

Die für die Phytotherapie eine der relevantesten Erkrankungen sind die Gallenwegsdyskinesien. Sie sind funktioneller Natur und beruhen auf einer Motilitätsstörung der Gallenblase und Gallengänge. Die hier wirksamen Heilpflanzen sind aber nicht isoliert auf die Gallenblase und die Gallengänge beschränkt zu sehen, sondern in aller Regel Ausdruck eines gestörten $\mathrm{Zu}$ sammenspiels aller Oberbauchorgane. Gerade bei diesen Erkrankungen kommt die Vielseitigkeit der Phytotherapie zur Gel- tung. Heilpflanzen haben nie nur eine Wirkung auf ein Organ, sondern zeichnen sich durch ein ganzes Portfolio ihrer Wirkstoffgemische an organübergreifenden Effekten aus. Pflanzen, die bei Gallenwegsdyskinesien wirksam sind, sind dies häufig auch bei der funktionellen Dyspepsie (Reizmagen, Reizdarm). Im Rahmen dieser Arbeit können bei weitem nicht alle Heilpflanzen beschrieben werden, die es verdient hätten.

Folgende Pflanzen haben einen Bezug zu funktionellen Gallenbeschwerden:

- Wermut

- Schöllkraut

- Erdrauchkraut

- Rettich

\section{Wermut}

„Wermot ist för alles got“ sagt der Norddeutsche und hat Recht, wenn er ans üppige Essen denkt. Die alten Ägypter und die Griechen schätzten und verwendeten schon das Kraut gegen viele Krankheiten, so steht es schon im berühmten Papyrus Ebers.

Im Paris des 19. Jahrhunderts kannte man die „grüne Fee“ und bekannte Literaten und Künstler waren ihr zur „L'heure verte“ erlegen. Auch mancher Seefahrer ging am Absinthismus elendig zu Grunde. Es geht um den Wermut, der bittere Beifuß (Artemisia absinthium) ein Korbblütler. Der Name verrät schon etwas von seinen Wirkstoffen: ein Bitterstoff (Amarum aromaticum). „Was bitter im Mund ist dem Magen gesund“ wussten immer schon die Pflanzenheilkundigen. So regt er den Appetit an durch die Erhöhung der Speichel- und Magensaftsekretion, wirkt verdauungsfördernd, karminativ und v.a. choleretisch. Auch besitzt er einen zentral anregenden, psychedelischen, visionsfördernden halluzinogenen Effekt. Es heißt, dass eine Tasse Wermuttee die Konzentration mehr fördert, als eine Tasse Kaffee.

\section{Monografie der Kommission E}

In der Monografie der Kommission E werden als Anwendungsgebiete Appetitlosigkeit, dyspeptische Beschwerden, die auf einer gestörten Galleausscheidung durch die Gallenblase beruhen, sowie Dyskinesien der Gallenwege angegeben.

\section{Tee und Tinktur}

Als Anwendungen dienen der Tee und die Tinktur

Tee: 1 Teelöffel fein geschnittener Droge mit 1 Tasse kochendem Wasser übergießen, 1-2, max. 5 Minuten ziehen lassen, abseihen; einige Zeit nach den Mahlzeiten je 1 Tasse trinken.

Tinktur: 3-mal täglich 10-30 Tropfen wegen der Bitterstoffe in reichlich Wasser einnehmen.

\section{Fertigpräparate}

Bewährte Fertigpräparate sind:

- Abdomilon ${ }^{\circledR}$ N Sirup (Fa. Cesra), 3-mal täglich 1 Messbecher.

- Amara-Tropfen (Fa. Weleda), bei Appetitlosigkeit 10-15 Tropfen 15 Minuten vor dem Essen einnehmen. Bei Störungen der Verdauung 10-15Tropfen 1 Stunde nach dem Essen einnehmen Kinder von 6-11 Jahre erhalten 5-8 Tropfen; Kleinkinder von 1-5Jahren 3-5 Tropfen täglich.

- metamarianum ${ }^{\circledR}$ B12 N Mischung (Fa. meta Fackler), 1- bis 6-mal täglich 5 Tropfen.

- Pascopankreat ${ }^{\circledR}$ Tabletten Filmtabletten (Fa. Pascoe), mittags und abends jeweils 1 gelbe Tablette vor und 1 rote Tablette während oder nach den Mahlzeiten unzerkaut mit etwas Flüssigkeit einnehmen.

Sämtliche genannten Präparate sind Komplexmittel und enthalten noch weitere synergistisch wirkende Pflanzenextrakte.

\section{Schölllkraut}

Das Schöllkraut (Chelidonium majus) enthält ca. 30 Alkaloide, Flavone, Bitterstoffe und ätherisches Öl. Es gehört zu den Mohngewächsen (Papaveraceae). Verwendet wird das blühende Kraut (Chelidonii herba). Ähnlich wie das Papaverin, aber schwächer, wirkt es spasmolytisch und choleretisch. Im Tierversuch zeigte sich eine Steigerung der Gallenproduktion von $60 \%$.

\section{Tee und Tinktur}

Angewendet wird Schöllkraut als Tee oder Tinktur.

Tee: 2 Teelöffel fein geschnittener Droge mit 1 Tasse kochendem Wasser übergießen, 5-10 Minuten ziehen lassen, absei- 
hen und zwischen den Mahlzeiten 3-mal

täglich 1 Tasse warm trinken.

Tinktur: 3-mal täglich 20 Tropfen in etwas Wasser.

\section{Fertigpräparate}

Geeignete Fertigpräparate sind:

- Chelidonium comp. Dilution (Fa. Weleda), 2- bis 3-mal täglich vor den Mahlzeiten einnehmen. Einzeldosis: Erwachsene und Kinder ab 6 Jahren 5-10Tropfen; Kinder von 1-5 Jahren 5-8 Tropfen; Säuglinge im 1.Jahr 3-5 Tropfen.

- Iberogast $^{\circledR}$ Flüssigkeit (Fa. Steigerwald), 3-mal täglich vor oder zu den Mahlzeiten in etwas Flüssigkeiten einnehmen; Erwachsene und Jugendliche ab 13 Jahren 20 Tropfen, Kinder von 6-12 Jahren 15 Tropfen, Kinder von 3-6Jahren 10Tropfen.

Auch diese Präparate sind als Komplexmittel im Handel.
Erdrauch

Hexen und Zauberer lösten sich durch ihn in Rauch auf: Ob daher der Name Erdrauch (Fumaria officinalis) stammt, ist eher ungewiss. Die Pflanze gehört ebenfalls zu den Mohngewächsen. Auch er hat u.a. diverse Alkaloide, die ähnlich wirken, wie die des Schöllkrauts. Dazu kommen u.a. Flavonoide und Bitterstoffe. Verwendet wird das Kraut. Er ist eine alte Heilpflanze, die allerdings heute kaum noch Verwendung findet.

\section{Tee}

Tee: 2 Teelöffel der zerkleinerten Droge mit 1 Tasse kochendem Wasser übergießen, 10 Minuten ziehen lassen, abseihen und zu den Mahlzeiten je 1 Tasse täglich warm trinken.

Pflanzliche Fertigpräparate sind, soweit beurteilbar, nicht mehr auf dem Markt.

\section{Rettich}

Der Rettich ist eine sehr alte, aus Asien stammende Kulturpflanze. Schon im alten
Ägypten gab man den Pyramidenbauern Rettich, Knoblauch und Zwiebeln, denn bei den schlechten hygienischen Verhältnissen brauchten die schwer schuftenden Arbeiter antibiotisch und gastrointestinal wirksame „Medikamente“. Das hat sogar zu dem möglicherweise ersten verbrieften Streik in der Geschichte geführt. Der Rettich gehört zu den Kreuzblütlern, will heißen Senfölpflanzen. Er enthält Vitamin C, Carotin, B-Vitamine, viele Mineralstoffe und Spurenelemente wie Kalium, Natrium, Magnesium, Kalzium, Phosphor, Eisen, Selen, Bor, Brom, Fluor, Kobalt, Mangan, Schwefel, Zink und Enzyme, Raphanol, Senfölglykoside und Bitterstoffe. So hat er im Gastrointestinaltrakt eine choleretische, spasmolytische, motilitätsfördernde, antimikrobielle, sogar antikanzerogene Wirkung. Er wirkt also u.a. bei dyspeptischen Beschwerden, besonders infolge von Dyskinesien der Gallenwege. 


\section{Einnahmeempfehlung}

Den Rettich (Abb. 3) nimmt man am besten als Frischpflanzensaft zu sich. Folgende Zubereitung hat sich bewährt:

Bei Gallen- und Lebererkrankungen nimmt man $50 \mathrm{ml}$ täglich auf nüchternen Magen ein, steigert die Menge nach einigen Tagen langsam auf bis zu 4-mal $200 \mathrm{ml}$ Rettichsaft täglich und reduziert dann die Menge wieder langsam auf $50 \mathrm{ml}$ täglich. Bei kurmäßiger Anwendung über 3 Wochen sollte man nach 4-5 Tagen eine Pause von 2-3 Tagen machen. Wer sich den Saft nicht selber herstellen möchte, was sehr einfach geht und preiswert ist, kann sich von der Firma Schoenenberger „Naturreiner Heilpflanzensaft Schwarzrettich Presssaft" besorgen.

Dosierung: Erwachsene und Kinder ab 12 Jahren 3-mal täglich vor den Mahlzeiten $10 \mathrm{ml}$ unverdünnt oder mit etwas Flüssigkeit einnehmen.

\section{Löwenzahn}

Der viel gescholtene Löwenzahn, den niemand im Garten haben möchte, ist alles andere als ein Unkraut, sondern eine der wertvollsten Heilpflanzen! Er ist ein Korbblütler und gehört zu den Zichoriengewächsen. Seine Inhaltsstoffe sind:

Bitterstoffe (z.B. Taraxin), Triterpenoide, Phytosterine, Inulin 40\%, Cholin, Vitamine (u.a. Ascorbinsäure), Mineralstoffe (u.a. viel Kalium).

Er hat gleich zwei wertvolle Wirkungen, nämlich auf den Verdauungstrakt und auf die Nieren. Im Gastrointestinaltrakt zeigt er seine cholagogen, verdauungsfördernden, appetitanregenden Eigenschaften, im Harnwegsbereich seine diuretisch aquaretische Wirkung und die ist durchaus nicht unbeträchtlich (deshalb nennt ihn der Franzose auch „piss en lit“). So eignet sich ein Löwenzahnextrakt v.a. bei dyspeptischen Beschwerden, bei Nierenschwäche, zur Frühjahrskur und wegen seines Bitterstoffgehaltes auch als bekömmlicher allerdings gewöhnungsbedürftiger Kaffeeersatz (Muckefuck).

Tee

Am besten wirkt er als Tee v.a. aus der frisch geernteten Pflanze, die man kurmäßig anwenden kann.

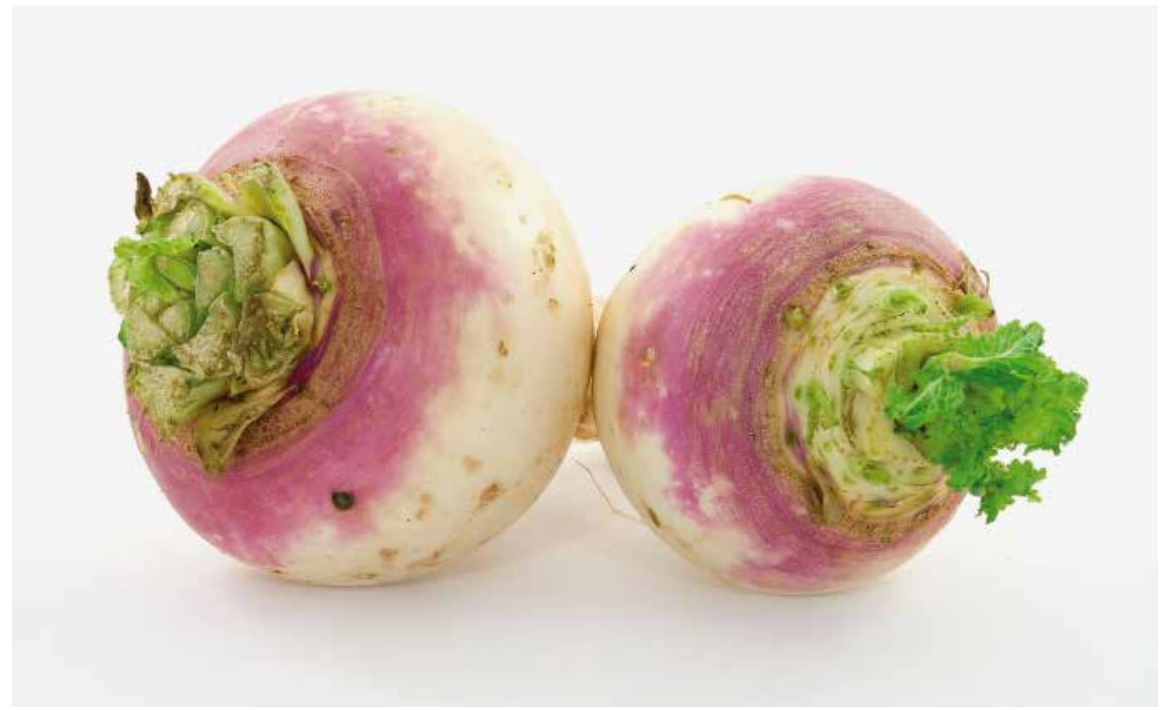

Abb. 3 Rettich. (C) dinostock/Fotolia

So wird der Tee zubereitet: 1-2 Teelöffel fein geschnittener Droge mit 1 Tasse Wasser kalt ansetzen, kurz aufkochen, übergießen, 15 Minuten ziehen lassen, abseihen; morgens und abends je 1 Tasse über 4-6 Wochen trinken.

\section{Tinktur}

Auch die Löwenzahntinktur kann sehr hilfreich sein: Man nimmt davon 3-mal täglich 10-15 Tropfen in etwas Wasser.

\section{Fertigpräparate}

Löwenzahnextrakte sind in diversen pflanzlichen und homöopathischen Kombinationsmitteln enthalten, die z.T. oben schon erwähnt wurden. Weiteres Präparat:

- Aranicyn Leber-Gallemittel Mischung (Fa. Weber \& Weber), Dosierung: Bei akuten Zuständen alle halbe bis ganze Stunde, höchstens 6-mal täglich, je 5 Tropfen, bei chronischen Verlaufsformen 1- bis 3-mal täglich 5 Tropfen einnehmen.

\section{Fazit}

Die Phytotherapie ist eine starke und verlässliche Partnerin in der Behandlung der Leber- und Gallenerkrankungen, wenn man sie gezielt einsetzt und sie in ein sinnvolles Therapiekonzept einfügt.
Online zu finden unter:

http://dx.doi.org//10.1055/s-0041-103869

Interessenkonflikte: Der Autor erklärt, dass keine wirtschaftlichen oder persönlichen Verbindungen bestehen.

\section{ÜBER DEN AUTOR}

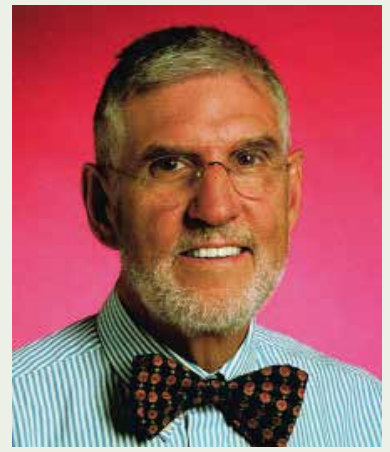

Dr. med. Hartmut Dorstewitz ist Facharzt für Allgemeinmedizin, Naturheilverfahren, Homöopathie sowie Dozent für Biologische Medizin (Universität Mailand/WHO). 2003-2007 Präsident des Deutschen Naturheilbundes e.V., Ehrenvorsitzender des Arbeitskreises für Mikrobiologische Therapie e.V., Lehrtätigkeit für Phytotherapie für den Kneipp-Ärztebund Bad Wörishofen.

KORRESPONDENZADRESSE

Dr. med. Hartmut Dorstewitz

Alpenstraße 5

85614 Kirchseeon

E-Mail: dowiki@gmx.de 